\title{
Smoking and potentially preventable hospitalisation: The benefit of smoking cessation in older ages
}

\author{
Bich Tran ${ }^{\mathrm{a}, *}$, Michael O. Falster ${ }^{\mathrm{a}}$, Kirsty Douglas ${ }^{\mathrm{b}}$, Fiona Blyth ${ }^{\mathrm{c}, \mathrm{d}}$, Louisa R. Jorm ${ }^{\mathrm{a}}$ \\ a Centre for Big Data Research in Health, Faculty of Medicine, UNSW Australia, NSW 2052, Australia \\ ${ }^{\mathrm{b}}$ Australian National University, Canberra ACT 0200, Australia \\ c The Sax Institute, Haymarket, NSW 2000, Australia \\ d Concord Clinical School, University of Sydney, NSW 2006, Australia
}

\section{A R T I C L E I N F O}

\section{Article history:}

Received 21 October 2014

Received in revised form 13 February 2015

Accepted 14 February 2015

Available online 4 March 2015

\section{Keywords:}

Smoking

Preventable hospitalisation

Rate advancement period

45 and Up Study

\begin{abstract}
A B S T R A C T
Aims: Reducing preventable hospitalisation is a priority for health systems worldwide. This study sought to quantify the contribution of smoking to preventable hospitalisation in older adults and the potential benefits of smoking cessation.

Methods: Self-reported smoking data for 267,010 Australian men and women aged 45+ years linked with administrative hospital data were analysed using Cox's models to estimate the effects on risk of hospitalisation for congestive heart failure (CHF), diabetes complications, chronic obstructive pulmonary disease (COPD) and angina. The impacts of smoking and quitting smoking were also quantified using risk advancement periods (RAP).

Results: The cohort included 7\% current smokers, 36\% former smokers and 57\% never smokers. During an average follow-up of 2.7 years, $4 \%$ of participants had at least one hospitalisation for any of the study conditions ( $0.8 \%$ for CHF, $1.7 \%$ for diabetes complications, $0.8 \%$ for COPD and $1.4 \%$ for angina). Compared to never smokers, the adjusted hazard ratio for hospitalisation for any of the conditions for current smokers was 1.89 (95\% CI 1.75-2.03), and the RAP was 3.8 years. There were strong dose-response relationships between smoking duration, smoking intensity and cumulative smoking dose on hospitalisation risk. The excess risk of hospitalisation and RAP for COPD was reduced within 5 years of smoking cessation across all age groups, but risk reduction for other conditions was only observed after 15 years.

Conclusion: Smoking is associated with increased risk of preventable hospitalisation for chronic conditions in older adults and smoking cessation, even at older ages, reduces this risk.
\end{abstract}

(c) 2015 Elsevier Ireland Ltd. All rights reserved.

\section{Introduction}

Preventable hospitalisations are those that might be avoided through prevention and management in primary care, and rates of these admissions are used internationally as an indicator of health system performance (Jorm et al., 2012). Preventable hospitalisations account for around $10 \%$ of total hospital stays and total hospital expenditure (Stranges and Stocks, 2008), and reducing them is a priority for health systems worldwide (Muenchberger and Kendall, 2010).

The chronic conditions included in commonly used definitions of preventable hospitalisation include congestive heart failure (CHF), diabetes complications, chronic obstructive pulmonary disease (COPD) and angina, all of which are smoking-related.

\footnotetext{
* Corresponding author. Tel.: +612 93850504.

E-mail address: bich.tran@unsw.edu.au (B. Tran).
}

Accordingly, there is clear potential to reduce the rate of these hospitalisations through interventions to promote smoking cessation (Jackson et al., 2001). The majority of preventable hospitalisations for chronic conditions occur among people aged 65 years and over (Stranges and Stocks, 2008). While it has been clearly demonstrated that quitting smoking at age 60 years or older reduces the risk of mortality from all causes and many smoking-related causes (Gellert et al., 2012; He et al., 2014), few population-based studies have quantified the benefits of "late" quitting for preventable hospitalisation outcomes. Existing studies of the relationship between smoking and preventable hospitalisation in older populations have been restricted to specific patient groups (Godtfredsen et al., 2002; Shah et al., 2010), have presented combined mortality and morbidity endpoints (Gellert et al., 2013b), or have not stratified according to age at quitting (Baumeister et al., 2007).

In this study, data from a large prospective cohort of Australian men and women aged 45 years and over, linked with hospital morbidity data were used to: (1) quantify the effects of smoking on 
risk of preventable hospitalisation (expressed both as hazard ratios [HRs] and risk advancement periods [RAP]) for CHF, diabetes complications, COPD and angina; (2) investigate the contributions of smoking duration and smoking intensity to these risks; and (3) investigate the impact of quitting smoking at older ages on risk of preventable hospitalisation.

\section{Methods}

\subsection{Participants}

This analysis was part of the Assessing Preventable Hospitalisation InDicators (APHID) study (Jorm et al., 2012). APHID uses linked survey and administrative data for participants in the Sax Institute's 45 and Up Study, a prospective cohort of 267,091 men and women aged 45 years and over and resident in New South Wales (NSW), Australia (Banks et al., 2008). Participants were randomly sampled from the database of the national health insurance scheme (Medicare Australia). Participants entered the study by completing a mailed self-administered questionnaire at study entry (between February, 2006 and April, 2009) and providing written consent for long-term follow-up and linkage of their health information to a range of routine health databases. People residing in non-urban areas and those aged 80 years and over were oversampled. The overall response rate for the 45 and Up Study is estimated to be $18 \%$ and the study included about $10 \%$ of the NSW population aged 45 and over.

\subsection{Data collection}

2.2.1. Social-demographic data. Exposure and confounding variables used in this analysis were derived from self-reported data from the 45 and Up Study baseline questionnaire collected at study entry (available at https://www.saxinstitute. org.au/our-work/45-up-study/), apart from the measure of remoteness of residence, which was assigned according to the mean score of Accessibility Remoteness Index of Australia Plus (ARIA+) for the Postal Area of the participant's address (AIHW, 2004)

Socio-demographic data included participants' educational level (did not complete high school, high school or equivalent, University or higher), marital status (single, married or partnered, windowed or separated), language spoken at home (English, language other than English), annual household income $(<\$ 10,000$, $\$ 10,000-\$ 29,999, \$ 30,000-\$ 49,999, \$ 50,000-\$ 69,999, \$ 70,000$ or more, and II would rather not answer the question'), and health insurance status (private health insurance with or without extras, Department of Veterans Affairs card, health care card, and none). Participants' self-reported weight $(\mathrm{kg})$ and height $(\mathrm{cm})$ without shoes were used to calculate body mass index $\left(\mathrm{BMI}, \mathrm{kg} / \mathrm{m}^{2}\right)$, which was classified into groups according to WHO categorisation: 'underweight' $\leq 18.5 \mathrm{~kg} / \mathrm{m}^{2}$, 'healthy weight' $<18.5-25 \mathrm{~kg} / \mathrm{m}^{2}$, 'overweight' $25.1-30 \mathrm{~kg} / \mathrm{m}^{2}$, and 'obese' $>30 \mathrm{~kg} / \mathrm{m}^{2}$.

A score was generated for participants' number of positive health behaviours based on meeting recommendations for five behaviours (less than 14 alcohol drinks per week, more than $2.5 \mathrm{~h}$ of intensity-weighted physical activity over at least 5 sessions per week, at least 2 servings of fruit and 5 servings of vegetables per day, less than $8 \mathrm{~h}$ of sitting and not less than $7 \mathrm{~h}$ sleeping time per day) (Tran et al., 2014).

2.2.2. Smoking history and derivation of smoking variables. Current smoking status was based on responses to the questions "Have you ever been a regular smoker?", and (if yes) "Are you a regular smoker now?" Current and former smokers were asked further detailed questions about their smoking history, including the age at which they started smoking regularly, the age at which they stopped smoking regularly (for former smokers) and the average number of cigarettes or pipes/cigars they smoked each day.

Smoking duration was defined as the difference between starting age and either quitting age (former smokers) or current age (current smokers). Smoking intensity was calculated as the number of cigarettes and pipes/cigars smoked each day. Cumulative number of pack-years of tobacco exposure was derived by dividing the number of cigarettes and pipes/cigars smokes average each day by 20 and multiplying by the total number of years smoked. Time since quitting smoking was calculated as the difference between the age at which former smokers had stopped smoking regularly and their current age. For categorical analyses, each of the above smoking measures was categorised into quartiles. Participants with missing smoking data were excluded from the analyses.

2.2.3. Outcomes. Incident preventable hospitalisations for CHF, diabetes complications, COPD and angina were ascertained using linked hospital morbidity data, which captures all separations from public and private sector hospitals in NSW, based on the ICD10-AM diagnosis codes specified in the 2012 Australian National Healthcare Agreement potentially preventable hospitalisation indicator (NHA, 2012). Hospital morbidity data and death registration records were linked to the baseline data from the 45 and Up Study by the Centre for Health Record Linkage (http://www.cherel.org.au/) using probabilistic record linkage methods and commercially available software.
2.2.4. Statistical analysis. Participants were followed from the date of recruitment to either the date of first hospitalisation for each of the study conditions (CHF, diabetes complications, COPD and angina) or death or 30 December, 2010 (the last date to which hospital data were available), whichever occurred first. Separate analyses were run for each smoking variable (smoking status, smoking duration, smoking intensity, cumulative smoking dose and time since quitting smoking) and for each condition.

All participants were included in analyses of the association between smoking and preventable hospitalisation, with never-smokers as the reference group, except for analyses of time since quitting, where current smokers were used as the reference group. Cox proportional hazards models with age as the underlying time variable (Thiebaut and Benichou, 2004) were used to estimate hazard ratios (HRs) and 95\% confidence intervals (CIs) for risk of hospitalisation. To assess the dose-response relationship between smoking variables and risk of hospitalisation, smoking duration (per 10 years), smoking intensity (per 10 cigarettes/day), cumulative dose (per 10 pack-years) or time since quitting (per 10 years) were included in models among ever-smokers only.

A number of variables were considered to influence both smoking and the risk of hospitalisation (Tran et al., 2014). In this analysis, we included age, sex, level of education, marital status, household income, speaking a language other than English at home, private health insurance status and remoteness of residence as social-demographic characteristics; and body mass index, number of positive health behaviours and prior admission in the 12 months prior to study entry as health and behavioural characteristics of the study participants. For present and former smokers who did not report the number of cigarettes smoked per day $(N=1475,1.3 \%$ of the population who reported ever smoking), median values for smoking intensity in the population were assigned ( 20 cigarettes per day for men and 15 cigarettes per day for women). This imputation had negligible impacts on the overall risk estimates.

Point estimates of risk advancement periods (RAP) for each smoking variable were derived from multivariable Cox models as the ratio of the regression coefficients for smoking exposure (by category) to the regression coefficient for age (as a continuous variable). Confidence intervals for RAP were calculated using variance and covariance estimates for regression coefficients (Brenner et al., 1993). Values of RAP describe how much sooner a given risk of hospitalisation is reached among exposed than among unexposed individuals: positive RAPs suggest the risk will be advanced to younger ages, whereas negative RAPs suggest the risk will be postponed to older ages (Liese et al., 2000).

To study the relative importance of smoking intensity and smoking duration on risk of hospitalisation, effect modification analysis was conducted for the effect of smoking intensity (median cut-off: 15 cigarettes per day) and duration of smoking (median cut-off: 25 years). RAPs for time since quitting smoking were also estimated according to strata of age at study entry ( $<65,65-74$ and $\geq 75$ years).

To assess the potential impact on our estimates of quitting smoking in response to a recent diagnosis of the study conditions or other smoking-related diseases ("sick-quitter bias"; Sargent et al., 2012), sensitivity analyses were performed that combined former smokers who had quit less than 5 years ago with current smokers

All analyses were performed using Stata 12.0. A significance level of $P<0.05$ was used for all comparisons.

\section{Results}

Of the 267,091 participants in the 45 and Up Study, 60 participants were excluded because of missing date of study entry and a further 21 were excluded because of possible inconsistent linkage, leaving 267,010 eligible participants included in this analysis. The mean age of participants was 63 years (standard deviation 11 years). Women comprised nearly $54 \%$ of the cohort.

Over an average of 2.7 years follow-up (interquartile range: 2.3-2.9 years), 11,035 (4.1\%) participants were admitted to hospital at least once for any of the study conditions $(0.8 \%$ for congestive heart failure, $1.7 \%$ for diabetes complications, $0.8 \%$ for COPD and $1.4 \%$ for angina) (Table 1 ). There were significant differences between participants who were hospitalised and those who were not in terms of age, gender, BMI, level of education, marital status, household income, private health insurance, number of positive health behaviours and a history of preventable hospitalisation for the same condition in the past 12 months (Table 1). Residents of remote areas were more likely than those living in metropolitan areas to be hospitalised for CHF or COPD; but this was not observed for diabetes complications or angina. People who spoke a language other than English were more likely than English-speakers to be admitted for CHF and diabetes complications, but not for COPD and angina (Table 1 ). 
Table 1

Characteristics of participants at study entry, and number of persons admitted for preventable hospitalisation during follow-up.

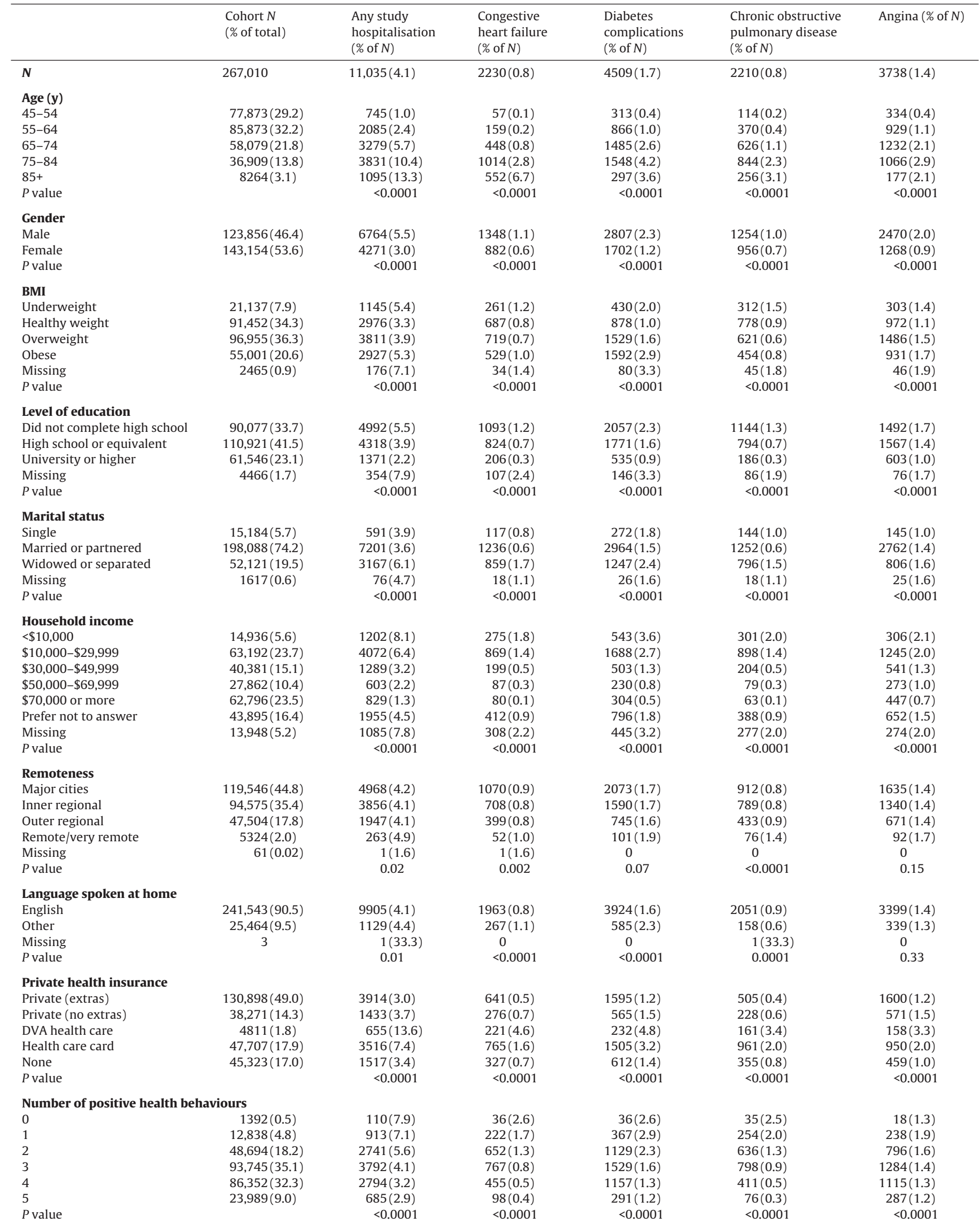


Table 1 (Continued)

\begin{tabular}{|c|c|c|c|c|c|c|}
\hline & $\begin{array}{l}\text { Cohort } N \\
\text { (\% of total) }\end{array}$ & $\begin{array}{l}\text { Any study } \\
\text { hospitalisation } \\
(\% \text { of } N)\end{array}$ & $\begin{array}{l}\text { Congestive } \\
\text { heart failure } \\
(\% \text { of } N)\end{array}$ & $\begin{array}{l}\text { Diabetes } \\
\text { complications } \\
(\% \text { of } N)\end{array}$ & $\begin{array}{l}\text { Chronic obstructive } \\
\text { pulmonary disease } \\
(\% \text { of } N)\end{array}$ & Angina $(\%$ of $N)$ \\
\hline \multicolumn{7}{|l|}{ Prior admission } \\
\hline No & $262,473(98.3)$ & $9354(3.6)$ & $2047(0.8)$ & $3718(1.4)$ & $1838(0.7)$ & $3472(1.3)$ \\
\hline Yes & 4537(1.7) & $1681(37.1)$ & $183(32.4)$ & $791(34.7)$ & $372(49.1)$ & $266(17.5)$ \\
\hline$P$ value & & $<0.0001$ & $<0.0001$ & $<0.0001$ & $<0.0001$ & $<0.0001$ \\
\hline \multicolumn{7}{|l|}{ Smoking status } \\
\hline Never smokers & $152,145(57.2)$ & $4871(3.2)$ & $1083(0.7)$ & $2153(1.4)$ & $467(0.3)$ & $1788(1.2)$ \\
\hline Former smokers & $94,585(35.6)$ & $5197(5.5)$ & $1026(1.1)$ & $2045(2.2)$ & $1345(1.4)$ & $1690(1.8)$ \\
\hline Current smokers & $19,194(7.2)$ & $893(4.7)$ & $102(0.5)$ & $284(1.5)$ & $373(1.9)$ & $239(1.3)$ \\
\hline
\end{tabular}

$P$ values were estimated excluding missing category.

Seven per cent of all participants were current smokers, $36 \%$ were former smokers and 57\% were never smokers (Table 1 ). Characteristics of participants at baseline by smoking status were shown in Supplementary Table S1. Current smokers (4.7\%) and former smokers $(5.5 \%)$ were significantly $(P<0.0001)$ more likely to be hospitalised for any of the study conditions than never smokers (3.2\%). Multivariable Cox regression showed that the greatest elevation in risk of hospitalisation among current smokers compared with never smokers was for COPD (HR 6.81, 95\% CI 5.87-7.89), with HRs for the other conditions ranging from 1.25 to 1.41 (Fig. 1 and Table S2). Risk advancement periods of 17.7, 6.7, 2.9 and 0.8 years were found among current smokers compared to never smokers for COPD, CHF, angina and diabetes complications, respectively (Table S2). There were clear dose-response relationships between smoking duration, intensity and cumulative smoking and increased risk of hospitalisation among people with history of smoking for any of the study conditions, and for each individual condition (Table S2).

Compared to current smokers, every 10 years increasing time since quitting smoking was associated with a $16 \%$ decreased risk of hospitalisation for any of the study condition s (HR 0.84, 95\% CI 0.82-0.86; Table S2). Analyses by time since quitting showed that smoking cessation for 5-14, 15-24 and 25 or more years was associated with a reduction in risk of hospitalisation for any of the study conditions of 3\%, 15\% and 40\%, respectively (Fig. 1 and Table S2). The corresponding RAPs were $-0.2,-1.8$ and -3.3 years (Table S2). The risk estimates for participants who had quit smoking less than 5 years ago were not significantly different to those for current smokers. Similar results were observed for each individual condition (Fig. 1 and Table S2).

Estimates of risk advancement periods (RAPs) for time since quitting smoking stratified by age at study entry are shown in Fig. 2.

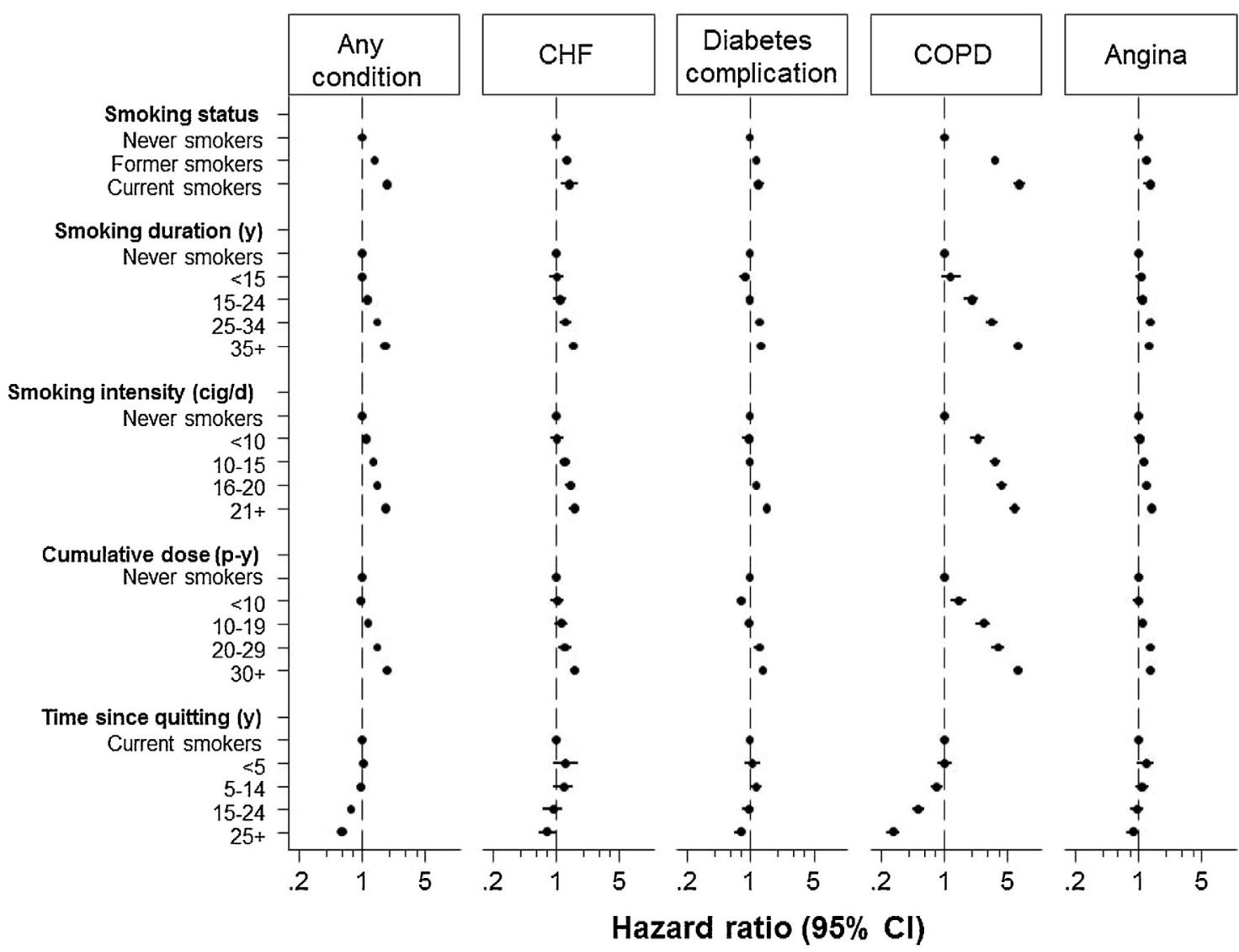

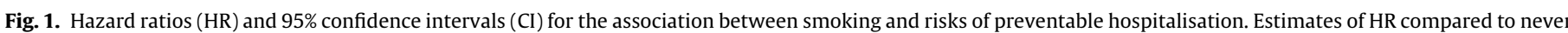

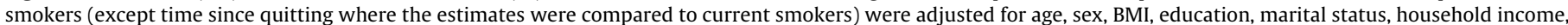

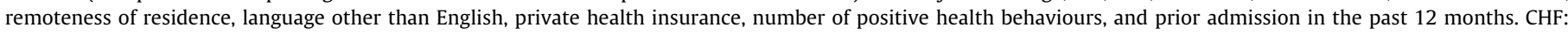
congestive heart failure; COPD: chronic obstructive pulmonary disease. 


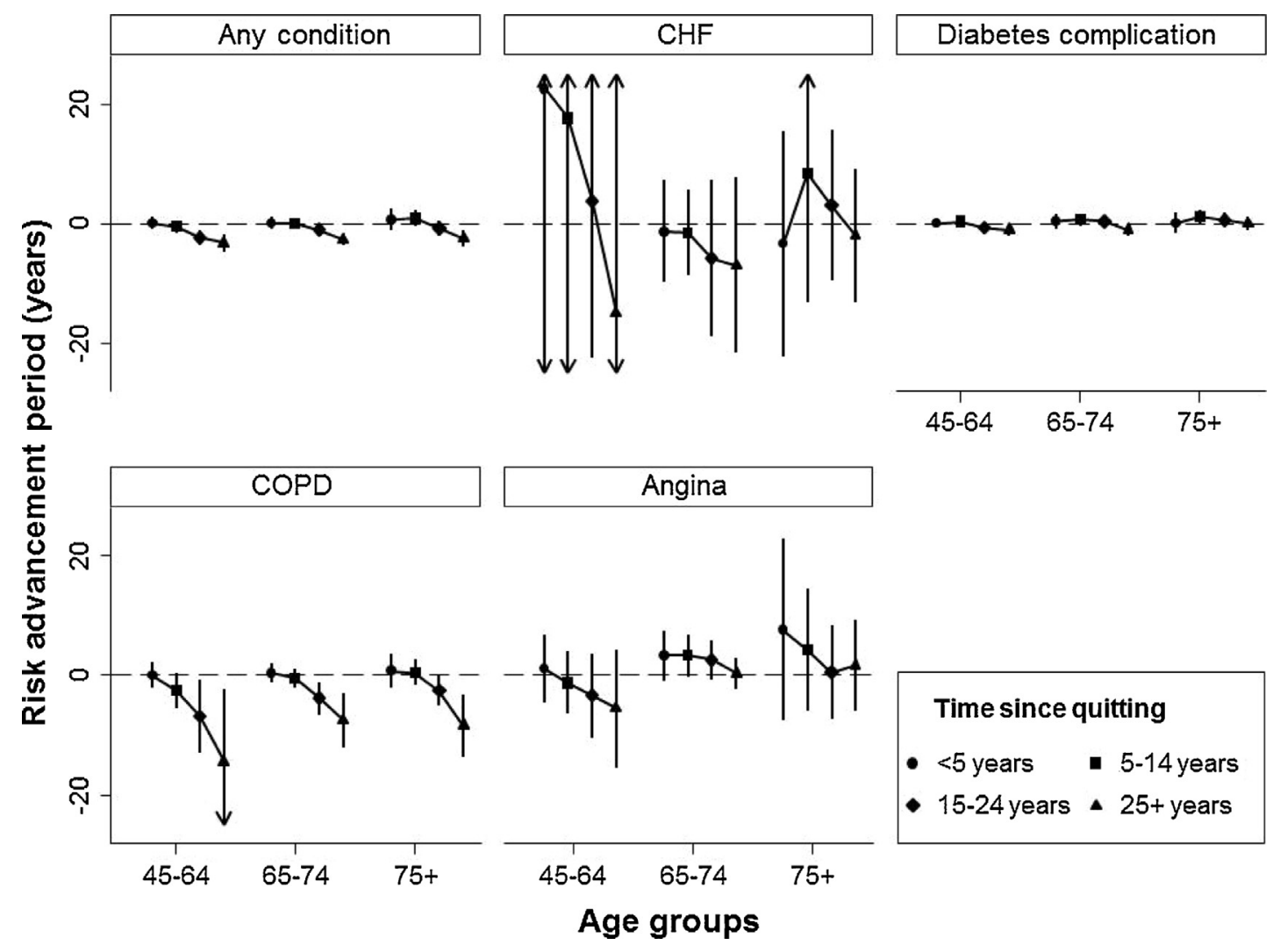

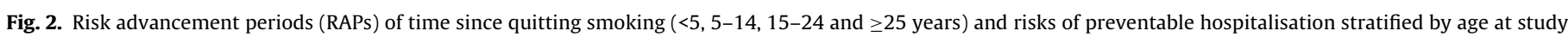

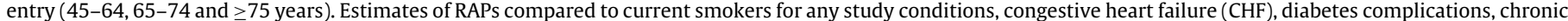

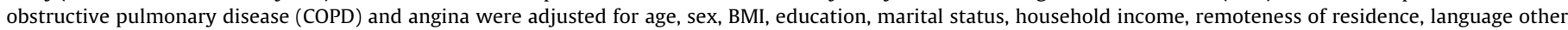
than English, private health insurance, number of positive health behaviours, and prior admission in the past 12 months.

Compared to current smokers, there was a decreasing trend in RAPs with time since quitting for any of the study conditions and for COPD. For CHF, diabetes complications and angina, the estimates of RAPs for time since quitting smoking were non-significant with wide confidence intervals across all age groups (Fig. 2).

Sensitivity analyses combining former smokers who had quit less than 5 years ago with current smokers did not significantly change the results (data not shown).

There was an additive effect of smoking duration and smoking intensity on risk of hospitalisation. Among those who had smoked for 25 years or longer, the risk of hospitalisation for any of the study conditions was significantly higher in those who smoked more than 15 cigarettes per day $(\mathrm{HR}=1.90,95 \% \mathrm{CI} 1.75-2.06)$, compared to those who smoked less than 15 cigarettes per day $(\mathrm{HR}=1.49,95 \% \mathrm{CI}$ 1.36-1.63; Table S3). Similar trends were observed for all individual conditions (Table S3).

\section{Discussion}

In this large population-based cohort of Australian men and women aged 45 years and over, we found that cigarette smoking substantially increased the risk of preventable hospitalisation for chronic conditions. Current smoking increased hospitalisation risk almost 7-fold for COPD, and by $25-41 \%$ for diabetes complications, angina and CHF. Prolonged smoking duration was associated with increased risk of hospitalisation, and this was exacerbated by heavy smoking intensity. For example, among smokers who had been smoking for 25 years or more, those who smoked more than 15 cigarettes a day had a more than 4 -fold increased risk of hospitalisation for COPD, compared with a 2.9-fold increased risk for those who smoked less than 15 cigarettes per day. Time since quitting smoking was associated with decreasing risk of hospitalisation and risk advancement periods (RAP), for the study conditions combined and for each individual condition. Quitting smoking at any age was associated with reduced RAPs for the study conditions combined and for COPD. The excess risk of hospitalisation and RAP for COPD was reduced within 5 years of smoking, but risk reduction for other conditions was only observed after 15 years.

To the best of our knowledge, this was the first investigation to examine the effect of time since quitting smoking and hospitalisations for a range of chronic diseases that are included in the commonly used rubric of 'preventable' hospitalisation, and to provide estimates of RAPs for these outcomes. Our results for factors related to smoking were broadly consistent with previous findings for specific patient cohorts including those with index admissions for COPD (Godtfredsen et al., 2002) and myocardial infarction (Shah et al., 2010). Our finding that the excess risk of preventable hospitalisation, especially for COPD, decreased among smokers who quit more than 5 years previously, even among older quitters, was also similar to findings from Germany with regard to excess risk of cardiovascular outcomes (Gellert et al., 2013b) and mortality (Gellert et al., 2013a). Taken together, these findings indicate that some of the smoking-related risk advancement for chronic disease can be reversed within a short time following cessation and that the beneficial effects of smoking cessation for some conditions are independent of age.

Patterns of association for other variables in our data with regard to the demographic characteristics of the population are consistent with literature, such as higher rates of preventable hospitalisation among men and older participants (Culler et al., 1998; Katteri et al., 2013). Similarly, the inverse associations between markers of socioeconomic status (Blustein et al., 1998; Katteri et al., 2013), 
such as income and education are also consistent with existing literature; as are the higher rates among remote residents (Ansari et al., 2012; Katteri et al., 2013) and those with fewer positive health behaviours (Russell et al., 2001; Tran et al., 2014). Although other Australian studies have reported that rates of hospitalisation overall, and of preventable hospitalisation (Correa-Velez et al., 2007; Singh and de Looper, 2002), were lower in overseas-born groups, these did not provide a detailed breakdown by individual condition. Our finding that rates of admission among people speaking a language other than English were higher for some conditions (congestive heart failure and diabetes complications) and lower or not different for others (COPD and angina) indicates that influences such as genetic predisposition, language skills and cultural background (Freund et al., 2013) may impact differently on various smoking-related conditions.

Our study had several strengths. Firstly, it used data from a large-scale cohort study that captured detailed data on several dimensions of smoking exposure, and a wide range of known and potential confounding and mediating factors. Secondly, the longitudinal design of the study with the independent ascertainment of incident hospitalisation helped to minimise the potential impact of recall bias. Finally, the inclusion of large numbers of older adults allowed precise estimation of age-specific risks and RAPs, and therefore of the potential benefits of quitting smoking even among the very old.

Nevertheless, this analysis also had some limitations. The use of self-reported data could result in potential misclassification of smoking exposure, and measures of changes in smoking behaviour, such as smoking intensity, over time were not available. The 45 and Up Study had a low response rate (18\%), reflecting the trend towards declining participation rates in cohort studies and other epidemiological studies (Morton et al., 2006). However, this response rate was greater than that achieved by the UK Biobank (5-10\%), a comparable contemporary cohort study which like the 45 and Up Study had a focus on achieving a large, diverse participant base for valid estimation of risk associations (Manolio et al., 2012).

Consistent with a "healthy cohort effect", the prevalence of current smoking (7\%) in the 45 and Up Study was lower than that reported in the NSW Health Survey (12\%), the most comparable population survey, which had a response rate of $60 \%$ (Barr et al., 2008). Although the possibility of bias cannot be ruled out, previous analysis showed that risk estimates for a range of exposure-outcome relationships calculated from the 45 and Study were consistent with those calculated from the NSW Health Survey (Mealing et al., 2010).

Although a number of potential confounders were controlled for in the analyses, the possibility of residual confounding by imperfect measurement or unmeasured confounders cannot be ruled out. The sensitivity analyses combining recent quitters with current smokers produced similar results to the main analyses, suggesting that the effects of quitting in response to recent disease diagnosis did not substantially bias the risk estimates downwards.

In summary, findings showed that smoking was associated with increased risk of preventable hospitalisation for CHF, diabetes complications, COPD and angina in people aged 45 years and over, and there were dose-response relationships between smoking intensity, smoking duration and cumulative smoking and these risks. The excess risk of preventable hospitalisations from these causes combined, and specifically for COPD, was reduced within 5-14 years by quitting smoking even at older ages. These findings indicate that promotion of smoking cessation is a key mechanism whereby primary care services, and the prevention system more broadly, can act to reduce preventable hospitalisation for chronic disease and the consequent health system and societal costs.

\section{Role of funding source}

The study was funded by a National Health and Medical Research Council Partnership Project Grant (\#1036858) and by partner agencies the Australian Commission on Safety and Quality in Health Care, the Agency for Clinical Innovation and the NSW Bureau of Health Information. The funders play no role in approving the publications.

\section{Contributors}

BT conducted the data analyses and drafted the manuscript. MF extracted data for analysis, assisted with statistical methods and data presentation, and reviewed and edited the manuscript. LJ conceived, designed and managed the study, obtained funding, reviewed and edited the manuscript and provided overall supervision. All authors reviewed and approved the final manuscript.

\section{Conflict of interest}

The authors have declared that no competing interests exist.

\section{Acknowledgements}

The APHID investigator team comprises Louisa Jorm, Alastair Leyland, Fiona Blyth, Robert Elliot, Kirsty Douglas, Sally Redman, Marjon van der Pol, Michael Falster, Bich Tran, Neville Board, Danielle Butler, Douglas Lincoln, Sanja Lujic, Damilola Olajide, Deborah Randall, Kim Sutherland and Diane Watson.

This research was completed using data collected through the 45 and Up Study (www.saxinstitute.org.au). The 45 and Up Study is managed by the Sax Institute in collaboration with major partner Cancer Council NSW; and partners: the National Heart Foundation of Australia (NSW Division); NSW Ministry of Health; beyondblue; Ageing, Disability and Home Care, Department of Family and Community Services; the Australian Red Cross Blood Service; and UnitingCare Ageing. We thank the many thousands of people participating in the 45 and Up Study, the NSW Ministry of Health, Medicare Australia and the NSW Register of Births, Deaths and Marriages for allowing access to the data, and the Centre for Health Record Linkage for conducting the probabilistic linkage of records.

\section{Appendix A. Supplementary data}

Supplementary data associated with this article can be found, in the online version, at http://dx.doi.org/10.1016/j.drugalcdep. 2015.02.028.

\section{References}

AIHW, 2004. Australian Institute of Health and Welfare. Rural regional and Remote Health: A guide to remoteness classifications. 2004 for the Postal Area of the participant's residential address as recorded by Medicare, Canberra.

Ansari, Z., Haider, S.I., Ansari, H., de Gooyer, T., Sindall, C., 2012. Patient characteristics associated with hospitalisations for ambulatory care sensitive conditions in Victoria, Australia. BMC Health Serv. Res. 12, 475.

Banks, E., Redman, S., Jorm, L., Armstrong, B., Bauman, A., Beard, J., Beral, V., Byles, J., Corbett, S., Cumming, R., Harris, M., Sitas, F., Smith, W., Taylor, L., Wutzke, S., Lujic, S., 2008. Cohort profile: the 45 and up study. Int. J. Epidemiol. 37, 941-947.

Barr, M., Baker, D., Gorringe, M., Fritsche, L., 2008. NSW Population Health Survey: Description of Methods. NSW Department of Health, Sydney

Baumeister, S.E., Schumann, A., Meyer, C., John, U., Volzke, H., Alte, D., 2007. Effects of smoking cessation on health care use: is elevated risk of hospitalization among former smokers attributable to smoking-related morbidity? Drug Alcohol Depend. 88, 197-203.

Blustein, J., Hanson, K., Shea, S., 1998. Preventable hospitalizations and socioeconomic status. Health Aff. (Millwood) 17, 177-189.

Brenner, H., Gefeller, O., Greenland, S., 1993. Risk and rate advancement periods as measures of exposure impact on the occurrence of chronic diseases. Epidemiology 4, 229-236. 
Correa-Velez, I., Ansari, Z., Sundararajan, V., Brown, K., Gifford, S.M., 2007. A six-year descriptive analysis of hospitalisations for ambulatory care sensitive conditions among people born in refugee-source countries. Popul. Health Metr. 5, 9.

Culler, S.D., Parchman, M.L., Przybylski, M., 1998. Factors related to potentially preventable hospitalizations among the elderly. Med. Care 36, 804-817.

Freund, T., Campbell, S.M., Geissler, S., Kunz, C.U., Mahler, C., Peters-Klimm, F., Szecsenyi, J., 2013. Strategies for reducing potentially avoidable hospitalizations for ambulatory care-sensitive conditions. Ann. Fam. Med. 11, 363-370.

Gellert, C., Schottker, B., Brenner, H., 2012. Smoking and all-cause mortality in older people: systematic review and meta-analysis. Arch. Intern. Med. 172, 837-844.

Gellert, C., Schottker, B., Holleczek, B., Stegmaier, C., Muller, H., Brenner, H., 2013a. Using rate advancement periods for communicating the benefits of quitting smoking to older smokers. Tob. Control 22, 227-230.

Gellert, C., Schottker, B., Muller, H., Holleczek, B., Brenner, H., 2013b. Impact of smoking and quitting on cardiovascular outcomes and risk advancement periods among older adults. Eur. J. Epidemiol. 28, 649-658.

Godtfredsen, N.S., Vestbo, J., Osler, M., Prescott, E., 2002. Risk of hospital admission for COPD following smoking cessation and reduction: a Danish population study. Thorax 57, 967-972.

He, Y., Jiang, B., Li, L.S., Li, L.S., Sun, D.L., Wu, L., Liu, M., He, S.F., Liang, B.Q., Hu, F.B. Lam, T.H., 2014. Changes in smoking behavior and subsequent mortality risk during a 35-year follow-up of a cohort in Xi'an, China. Am. J. Epidemiol. 179, 1060-1070.

Jackson, G., Bobak, A., Chorlton, I., Fowler, G., Hall, R., Khimji, H., Matthews, H., Stapleton, J., Steele, C., Stillman, P., Sutherland, G., Swanton, R.H., 2001. Smoking cessation: a consensus statement with special reference to primary care. Int. J. Clin. Pract. 55, 385-392.

Jorm, L.R., Leyland, A.H., Blyth, F.M., Elliott, R.F., Douglas, K.M., Redman, S., Investigators, H.I.D., 2012. Assessing Preventable Hospitalisation InDicators (APHID): protocol for a data-linkage study using cohort study and administrative data. BMJ Open 2.

Katteri, R., Anikeeva, O., Butler, C., Brown, L., Smith, B., Bywood, P., 2013. Potentially Avoidable Hospitalisations In Australia: Causes For Hospitalisations And Primary Health Care Interventions. PHC RIS Policy Issue Review. Primary Health Care Research \& Information Service, Adelaide.

Liese, A.D., Hense, H.W., Brenner, H., Lowel, H., Keil, U., 2000. Assessing the impact of classical risk factors on myocardial infarction by rate advancement periods. Am. J. Epidemiol. 152, 884-888.
Manolio, T.A., Weis, B.K., Cowie, C.C., Hoover, R.N., Hudson, K., Kramer, B.S., Berg, C., Collins, R., Ewart, W., Gaziano, J.M., Hirschfeld, S., Marcus, P.M., Masys, D. McCarty, C.A., McLaughlin, J., Patel, A.V., Peakman, T., Pedersen, N.L., Schaefer, C. Scott, J.A., Sprosen, T., Walport, M., Collins, F.S., 2012. New model for large prospective studies: is there a better way? Am. J. Epidemiol. 175, 859-866.

Mealing, N.M., Banks, E., Jorm, L.R., Steel, D.G., Clements, M.S., Rogers, K.D., 2010 Investigation of relative risk estimates from studies of the same population with contrasting response rates and designs. BMC Med. Res. Methodol. 10, 26.

Morton, L.M., Cahill, J., Hartge, P., 2006. Reporting participation in epidemiologic studies: a survey of practice. Am. J. Epidemiol. 163, 197-203.

Muenchberger, H., Kendall, E., 2010. Predictors of preventable hospitalization in chronic disease: priorities for change. J. Public Health Policy 31, 150-163.

NHA, 2012. National Healthcare Agreement: PI 22-Selected Potentially Preventable Hospitalisations, 2012. Australian Institute of Health and Welfare, Available at: http://meteor.aihw.gov.au/content/index.phtml/itemId/443687

Russell, L.B., Teutsch, S.M., Kumar, R., Dey, A., Milan, E., 2001. Preventable smoking and exercise-related hospital admissions. A model based on the NHEFS. Am. J. Prev. Med. 20, 26-34.

Sargent, J.D., Demidenko, E., Malenka, D.J., Li, Z., Gohlke, H., Hanewinkel, R., 2012. Smoking restrictions and hospitalization for acute coronary events in Germany. Clin. Res. Cardiol. 101, 227-235.

Shah, A.M., Pfeffer, M.A., Hartley, L.H., Moye, L.A., Gersh, B.J., Rutherford, J.D., Lamas, G.A., Rouleau, J.L., Braunwald, E., Solomon, S.D., 2010. Risk of all-cause mortality, recurrent myocardial infarction, and heart failure hospitalization associated with smoking status following myocardial infarction with left ventricular dysfunction. Am. J. Cardiol. 106, 911-916.

Singh, M., de Looper, M., 2002. Australian Health Inequalities: 1 Birthplace. Bulletin no. 2. AIHW Cat. No. AUS 27. AIHW, Canberra.

Stranges, E., Stocks, C., 2008. Potentially Preventable Hospitalizations for Acute and Chronic Conditions. HCUP Statistical Brief \#99. November 2010 Agency for Healthcare Research and Quality, Rockville, MD, Available at: http://www.hcup-us.ahrq.gov/reports/statbriefs/sb99.pdf

Thiebaut, A.C., Benichou, J., 2004. Choice of time-scale in Cox's model analysis of epidemiologic cohort data: a simulation study. Stat. Med. 23, 3803-3820.

Tran, B., Falster, M.O., Douglas, K., Blyth, F., Jorm, L.R., 2014. Health behaviours and potentially preventable hospitalisation: a prospective study of older Australian adults. PLOS ONE 9, e93111. 